\title{
Internal Hernia After One Anastomosis Gastric Bypass (OAGB): Lessons Learned from a Retrospective Series of 3368 Consecutive Patients Undergoing OAGB with a Biliopancreatic Limb of $150 \mathrm{~cm}$
}

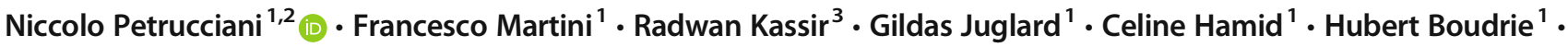 \\ Olivier Van Haverbeke ${ }^{1} \cdot$ Arnaud Liagre $^{1}$
}

Received: 13 November 2020 / Revised: 27 January 2021 / Accepted: 3 February 2021 / Published online: 8 April 2021

(C) The Author(s) 2021

\begin{abstract}
Background Internal hernia (IH) represents a relatively common and well-known complication after Roux-en-Y gastric bypass. $\mathrm{IH}$ after one anastomosis gastric bypass (OAGB) is less frequent and rarely reported in the literature. This study presents a series of IH after OAGB observed in a high-volume bariatric center.

Methods Data of patients who underwent OAGB with an afferent limb of $150 \mathrm{~cm}$ between May 2010 and September 2019 were prospectively collected and retrospectively analyzed. Data of patients undergoing surgery for IH during follow-up were collected and analyzed.

Results Ninety-six patients out of 3368 with a history of OAGB had intestinal incarceration in the Petersen's orifice (2.8\%). Specificity and sensitivity of computed tomography scans in the diagnosis of IH were $59 \%$ and $76 \%$, respectively. The mean timeframe between OAGB and surgery for IH was $21.9 \pm 18.3$ months. Mean body mass index at the time of IH surgery was $24.7 \pm 3.6$. Surgery was completed laparoscopically in $96.8 \%$ of cases. Nine patients $(9.3 \%)$ had signs of bowel hypovascularization. In all patients, the herniated bowel was repositioned, and the Petersen's orifice was closed, without the need for bowel resection. Mean hospital stay was $1.9 \pm 4.8$ days. The postoperative morbidity rate was $8.3 \%$. Long-term IH relapse was observed in 14 patients; signs of bowel hypovascularization due to incarceration in a small orifice was observed in eight of these patients (57\%).

Conclusions Incidence of IH after OAGB is $2.8 \%$. IH is associated with a low rate of bowel ischemia and the need for intestinal resection.
\end{abstract}

Keywords Bariatric surgery $\cdot$ One anastomosis gastric bypass $\cdot$ Internal hernia $\cdot$ Complications $\cdot$ Obesity

Niccolo Petrucciani

niccolo.petrucciani@uniroma1.it

Francesco Martini

framartini77@hotmail.com

Radwan Kassir

radwankassir42@hotmail.fr

Gildas Juglard

gildas.juglard@hotmail.fr

Celine Hamid

hamidceline@gmail.com

Hubert Boudrie

hboudrie@capio.fr
Olivier Van Haverbeke

drvanhaverbeke@hotmail.fr

Arnaud Liagre

arnaud.liagre@orange.fr

Bariatric Surgery Unit, Ramsay Générale de Santé, Clinique des Cedres, Cornebarrieu, France

2 Department of Medical and Surgical Sciences and Translational Medicine, Faculty of Medicine and Psychology, St Andrea Hospital, Sapienza University, via di Grottarossa 1035-9, 00189 Rome, Italy

3 Department of Digestive Surgery, CHU Félix Guyon, Saint Denis, La Réunion, France 


\section{Introduction}

Internal hernia $(\mathrm{IH})$ represents a relatively common and potentially serious complication after gastric bypass to treat morbid obesity [1]. After Roux-en-Y gastric bypass (RYGB), IH occurs mainly in the Petersen's and intermesenteric defect, and its incidence ranges from approximately 1 to $12 \%$ in different series [1-5]. Most patients have IH at the intermesenteric defect, whereas IH at the Petersen's is less common [1]. However, IH at the intermesenteric defect may be very dangerous due to the small size of the orifice. The herniation of the small bowel in mesenteric orifices created by the RYGB procedure may have severe consequences, including small bowel obstruction, ischemia, necrosis, and perforation if the diagnosis and treatment are not prompt [6]. It is mandatory to close the Petersen's and intermesenteric orifices during RYGB to reduce the incidence of IH. However, the closure of these defects may cause specific complications [7-10]. In mild cases, the IH may also cause chronic digestive symptoms, including pain, discomfort, and nausea [2]. IH is especially dangerous in pregnant women, with reported cases of maternal and fetal death due to bowel ischemia consequent to $\mathrm{IH}$ with late diagnosis and treatment $[11,12]$.

One anastomosis gastric bypass (OAGB) is a relatively recent bariatric procedure, which has rapidly gained acceptance and diffusion worldwide and represents $7.6 \%$ of all bariatric interventions. It is the third most frequent procedure following sleeve gastrectomy (SG) and RYGB $[13,14]$. Several authors have demonstrated the efficacy and safety of OAGB in treating obesity and its related comorbidities, and the procedure has been recognized by the International Federation for the Surgery of Obesity and Metabolic Disorders (IFSO) as a mainstream bariatric procedure [15-18].

IH occurs less frequently after OAGB than after RYGB. The first case was described in 2016 [19], as a bowel loop incarcerated behind the omega limbs, from the patient's left to right. IH after OAGB may have different clinical characteristics. However, only a few case reports, video reports, or small series have been published on this subject, therefore, little is known about IH after OAGB.

This study aims to present a series of IH after OAGB observed in a high-volume bariatric center, analyzing the diagnosis, treatment, and outcomes of this complication.

\section{Patients and Methods}

Between May 2010 and September 2019, 3368 patients underwent OAGB with a biliary limb of $150 \mathrm{~cm}$ and without closure of the Petersen's defect. The rationale is that the Petersen's orifice is usually large after OAGB and bowel strangulation with ischemia is rare. Starting from October 2019, the French National Authority of Health (Haute Autorité de Santé) removed OAGB from the list of reimbursable surgical procedures, therefore, bariatric surgeons in our institution stopped performing OAGB. Data of patients admitted for clinical suspicion of IH between May 2010 and May 2020 were collected and analyzed. Computed tomography (CT) scans with the ingestion of hydrosoluble contrast and injection of intravenous contrast was usually performed for preoperative diagnosis. These patients and patients with the incidental diagnosis of IH during cholecystectomy constitute our study group. Details of patients and disease characteristics were prospectively collected in a computerized database. Patients were retrieved from a prospectively maintained database of all bariatric procedures performed in our institution. The Institutional Review Board approved the present study, which is registered as IORG-IRB: IORG0009085 COS-RGDS-2019-11001-LIAGRE-A.

\section{Patient Management and Work-up}

All the patients included underwent OAGB with a biliopancreatic limb of $150 \mathrm{~cm}$ at our institution, as described previously [20]. The stomach was sectioned at the level of the incisura angularis and calibrated on a $36 \mathrm{Fr}$ bougie to fashion a long and narrow pouch. An antecolic laterolateral gastrojejunal anastomosis was created using a stapler with a $60-\mathrm{mm}$ vascular cartridge. No closure of mesenteric defects was performed. The postoperative follow-up of patients consisted of physical examination and blood tests every 6 months. Patients with acute abdominal pain were admitted to our emergency department and routinely assessed by a bariatric surgeon.

Patients presenting with acute abdominal pain were classified and managed as reported in Fig. 1. Patients presenting with epigastric and left hypochondrial pain underwent $\mathrm{CT}$ with oral contrast administration and intravenous contrast injection. If the CT scan was negative, upper GI endoscopy was performed. In cases where anastomotic ulcer was found without perforation, medical treatment was started. If IH was found, the patients underwent laparoscopic exploration. In patients with persistent abdominal pain, laparoscopic exploration was performed according to clinical evaluation.

\section{Radiological Evaluation (CT Scan with Oral Contrast Administration and Intravenous Contrast Injection)}

IH was suspected in case of one or more of these signs: swirl sign, strangulation of the superior mesenteric vein, engorged mesenteric vessels and edema, engorged lymph nodes, ascites, bowel ischemia, or edema, as reported previously [21, 22]. 
Fig. 1 Diagnostic algorithm in patients presenting with abdominal pain after one anastomosis gastric bypass.

OAGB, one anastomosis gastric bypass; $\mathrm{CT}$, computed tomography; GI, gastrointestinal

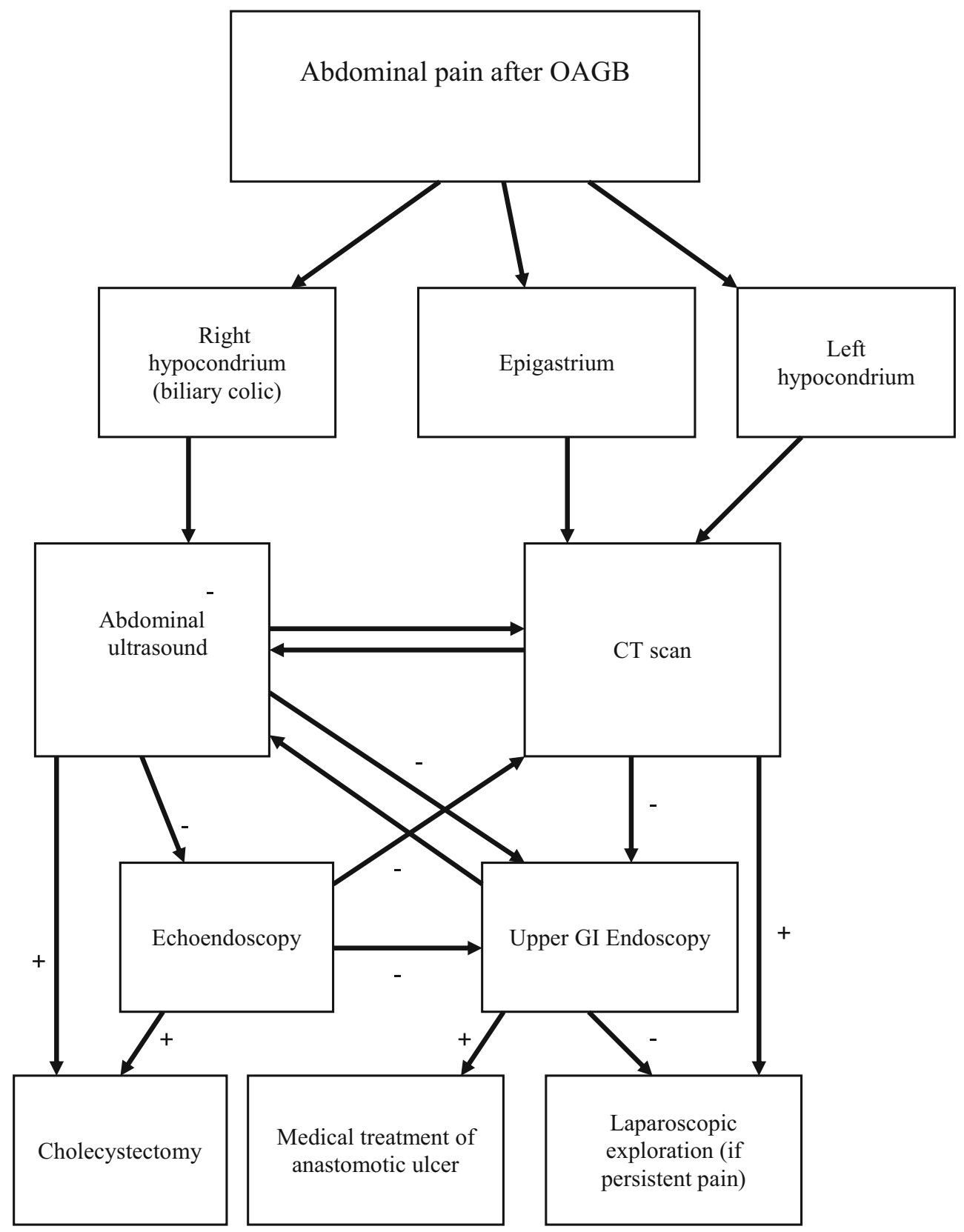

\section{Surgical Treatment}

In cases where there is suspicion of IH and a decision is made for surgical exploration, laparoscopy was performed. The patient was placed with the legs closed and both arms along the body, and the surgeon was at the patient's right. One trocar was placed in the left hypochondrium to lift the transverse mesocolon and open the Petersen's space and three more trocars were placed in the right abdomen for the camera and the two hands of the first surgeon. The small bowel was inspected totally starting from the ileocecal valve. The herniated content was repositioned and the mesenteric defect was closed using a running non-resorbable suture (Prolene 2/0).

\section{Postoperative Outcomes and Follow-up}

Postoperative complications were classified according to the Clavien-Dindo classification [23]. Early recurrence of internal hernia was defined as recurrence occurring in the first 30 days after surgery for internal hernia. Patients were examined one month after discharge to assess early postoperative complications. Then, they resumed their normal follow-up rate.

\section{Statistical Analysis}

Data were expressed as mean \pm standard deviation or median (range) or as numbers and percentages. 


\section{Results}

\section{Characteristics of Patients}

Overall, 136 patients were admitted for clinical suspicion of IH. Among these patients, 20 did not undergo a CT scan, three of them because they were pregnant, and 17 because they underwent explorative laparoscopy directly. The latter patients had experienced persistent episodes of pain and already had a negative abdominal ultrasound and GI endoscopy; therefore, a CT scan was not considered useful for diagnostic purposes (Fig. 2).

\section{Role of CT Scan}

In total, 116 patients underwent $\mathrm{CT}$ scans; signs of $\mathrm{IH}$ were retrieved in 77 patients. IH was confirmed in 63 of these cases, whereas no IH was found in 14 after surgical exploration. No signs of IH were observed in the CT scans of 39 patients but they underwent surgical exploration for persistent symptoms and high clinical suspicion; among them 19 had IH, the remaining 20 had a negative exploration (Fig. 2). The specificity and sensitivity of the CT scans were $59 \%$ and $76 \%$, respectively. The positive and negative predictive values of the CT scans were $82 \%$ and $51 \%$, respectively. Six other patients had incidental findings and treatment of IH during laparoscopic cholecystectomy for biliary colic.

\section{Characteristics of Patients Undergoing Surgery for IH}

\section{Clinical presentation}

Ninety-six patients out of 3368 with a history of OAGB had intestinal incarceration in the Petersen's orifice (2.8\%). The mean age was $38.3 \pm 11$ years (range: $19-71$ ) and 85 patients were female. The location of the abdominal pain was the right hypochondrium, the left hypochondrium, and the epigastrium in $14.5 \%, 34.5 \%$, and $51.2 \%$ of cases, respectively, and it was present for $20.9 \pm 33$ (range: 1-145) days. Body mass index (BMI) at the time of OAGB was $41.5 \pm 4.1 \mathrm{~kg} / \mathrm{m}^{2}$ (range: 35 56) and weight was $116.2 \pm 17.2 \mathrm{~kg}$ (range: $88-163$ ). Previous procedures included adjustable gastric banding (AGB) in two cases, SG in two cases, and AGB followed by SG in one case. The mean timeframe between OAGB and surgery for IH was

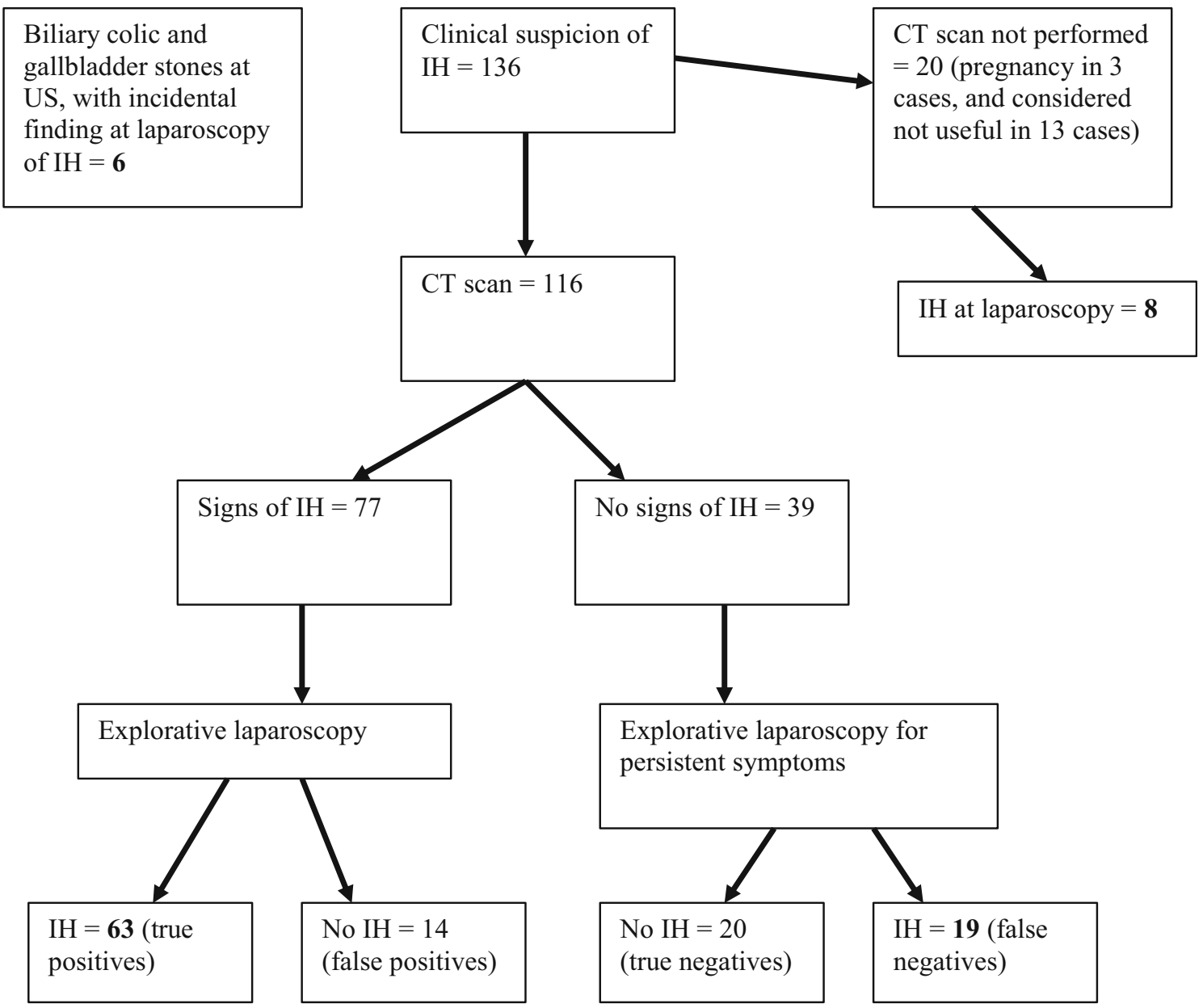

Fig. 2 Flowchart of the patients included. US, ultrasonography; CT, computed tomography; IH, internal hernia 
$21.9 \pm 18.3$ months (range: $3-88$ ). Mean weight and BMI at the time of IH surgery were, respectively, $69.4 \pm 12.6$ (range: 44-98), and $24.7 \pm 3.6$ (range: 18-33). Mean kilograms lost after OAGB were $47 \pm 12.5$ (range: $21-83$ ), and $\%$ excess weight loss (EWL) and \% total weight loss (TWL) were 104 \pm 22 (range: 50-156) and 40 \pm (range: 19-58), respectively. An abdominal CT scan with oral contrast administration was performed in 82 patients and was suggestive of IH in 63 (77\%). The findings of CT scans are shown in Table 1.

\section{Surgical Details}

Surgery was completed laparoscopically in 93 (96.8\%) cases and by laparotomy in three cases for difficult repositioning of the bowel during laparoscopy or because of the low laparoscopic experience of the operating surgeon. Nine patients (9.3\%) had signs of bowel hypovascularization in clinical (intense pain), radiological, or peroperative (venous stasis, abdominal fluid) examination. The herniated limb was the common limb in $74(77 \%)$ patients and the biliary limb in 22 (23\%). A $180^{\circ}$ torsion of the gastrojejunal anastomosis due to quasi-complete incarceration of the common limb was found in $47.3 \%$ of patients. The repositioning of the herniated bowel allowed the restoration of the correct positioning of the anastomosis. In all patients, the herniated bowel was repositioned and the Petersen's orifice was closed, without the need for bowel resections. Additional procedures performed are listed in Table 2.

\section{Postoperative short and long-term outcomes}

Mean hospital stay was $1.9 \pm 4.8$ days (range: $1-46$ ). The postoperative morbidity rate was $8.3 \%$ and mortality was null. Postoperative complications are reported in Table 3.

Long-term relapse of IH was observed in 14 patients (14.5\%); signs of bowel hypovascularization due to incarceration in a small orifice were found in eight of these patients $(57 \%)$. Four pregnant women underwent surgery without complications. Three of them were at 12-13 weeks of amenorrhea and underwent direct laparoscopic exploration, one patient at 23 weeks of amenorrhea underwent surgery after a

Table 1. Findings at CT scan

\begin{tabular}{ll}
\hline Finding & $N(\%)$ \\
\hline Mesenteric twist \pm mesenteric edema & $54(85.8 \%)$ \\
Intestinal invagination & $1(1.6 \%)$ \\
Perianastomotic inflammation & $1(1.6 \%)$ \\
Epiploic inflammation in right hypocondrium & $1(1.6 \%)$ \\
Small bowel obstruction & $6(9.4 \%)$ \\
\hline
\end{tabular}

CT computed tomography
Table 2. Additional procedures during surgery in a series of 96 patients who underwent surgical treatment of internal hernia after one anastomosis gastric bypass

\begin{tabular}{ll}
\hline & $N(\%)$ \\
\hline Braun anastomosis/conversion to RYGB & $1(1 \%)$ \\
T-tube positioning into a perforated ulcer & $1(1 \%)$ \\
Bowel suture following bowel iatrogenic injury & $1(1 \%)$ \\
Drain positioning & $1(1 \%)$ \\
Umbilical hernia repair & $1(1 \%)$ \\
Cholecystectomy & $13(13.5 \%)$ \\
Bowel resection & $0(0 \%)$ \\
\hline
\end{tabular}

RYGB Roux-en-Y gastric bypass

CT scan confirmed a diagnosis of IH. Six patients with a diagnosis of cholecystitis at ultrasound or CT scan underwent laparoscopy, and a concomitant IH was found. In cases with a double diagnosis, it was difficult to determine the etiology of the pain.

\section{Discussion}

IH after OAGB is not a well-known condition. We report in this paper the first series of patients treated for IH after OAGB. In 2016, Facchiano et al. reported the first single case of $\mathrm{IH}$ after OAGB [19], and since then only a few case reports have been published about the existence and potential danger of this condition [24-28]. In our institution, OAGB was adopted early and widely and we gathered extensive experience using a modified technique of OAGB with a biliary limb of $150 \mathrm{~cm}$ $[20,29,30]$. We could analyze the incidence of IH after OAGB without closure of the mesenteric defects on a series of 3368 patients. The incidence of IH (diagnosed at laparoscopy) was $2.8 \%$ (96 patients), which is lower than that reported after RYGB without closure of mesenteric defects $[8,31]$.

Symptoms of IH may be chronic and not specific, as seen for IH in patients with a history of RYGB. Differential diagnoses include gallbladder diseases, stone migration in the common bile duct, pancreatitis, anastomotic ulcer, gastritis or esophagitis, and small bowel obstruction due to postoperative adhesions. The diagnosis is mainly based on clinical suspicion after a CT scan, with low specificity and sensitivity compared with laparoscopic exploration $(59 \%$ and $76 \%$ in our patients, respectively), and on the exclusion of other possible diagnoses (mainly gallbladder disease and anastomotic ulcer). Laparoscopic exploration was offered also in patients with negative imaging but persistent symptoms suggestive of IH. Six patients with occasional diagnosis at laparoscopy were included. In these patients, the indication for surgery was symptomatic gallbladder lithiasis, but IH was found and repaired, due to the possibility that non-specific symptoms 

complications in a series of 96 patients who underwent surgery for an internal hernia after one anastomosis gastric bypass
Table 3. Postoperative

\begin{tabular}{lll}
\hline Complication & Number & Clavien-Dindo grading \\
\hline Wound infection & 1 & 1 \\
Hypertension & 1 & 2 \\
Pneumonia after inhalation & 2 & $4 \mathrm{a}$ \\
Peritonitis for bowel leak & 1 & $3 \mathrm{~b}$ \\
Precocious (within 30 days) relapse of IH & 2 & $3 \mathrm{~b}$ \\
Abdominal pain respondent to medical treatment & 1 & 1 \\
\hline
\end{tabular}

IH internal hernia

may have been related to bowel passage into the Petersen's orifice. We recommend systematic exploration of the Petersen's orifice in patients undergoing laparoscopy for abdominal pain, even if preoperatively another diagnosis was suspected (e.g., biliary colic).

Surgical treatment consists of the closure of the Petersen's orifice with a non-resorbable suture and is associated with a very low morbidity rate and a short mean hospital stay. Laparoscopy was possible in more than $96 \%$ of patients. No cases of bowel ischemia requiring intestinal resection were observed. This is probably related to the large mesenteric defect created by the OAGB, allowing bowel incarceration to strangulate the bowel. In $47.3 \%$ of patients, we observed at laparoscopy a $180^{\circ}$ torsion of the gastrojejunal anastomosis due to quasi-complete incarceration of the common limb. This observation is peculiar to OAGB: only a single point of fixation exists for the presence of a single anastomosis and the weight of the herniated bowel may easily cause rotation of the anastomosis. This phenomenon is never observed in the case of IH after RYGB, where the two anastomoses represent two points of fixation. It should be highlighted that the authors experienced a higher risk of bowel ischemia in patients with recurrent IH after OAGB. In those patients, the Petersen's orifice was only partially open and so it was smaller, leading to a higher rate of bowel ischemia, which was present in eight out of 14 patients. In our experience, more than one recurrence may be possible in the same patient during time.

No clear recommendations exist on the closure of Petersen's orifice during OAGB. This study identifies the rate of secondary surgery to treat an IH in a large series of patients, and the associated short and long-term outcomes. According to our experience, IH after OAGB is rarely associated with serious complications such as bowel ischemia.

We highlight several important points. The organization and timing of patients' treatment are very important to avoid wasting time and to reduce the incidence of bowel necrosis. Our patients are instructed about potentially dangerous symptoms and are encouraged to directly consult the bariatric surgical unit, which guarantees rapid and expert management. The referral of patients to centers lacking experience in bariatric surgery may be responsible for diagnostic and treatment delay [11]. Furthermore, in our opinion, the experience of the center and the number of bariatric surgeries per year is a second important factor. We believe that this is one of the factors permitting no cases of bowel necrosis out of 3368 OAGB in our institution.

The advantage of closure of the Petersen's space is the theoretical diminution of the incidence of IH (which however has not been proven after OAGB, whereas it has been demonstrated after RYGB). Disadvantages of the closure are (1) the creation of a smaller space in case of incomplete or failing of the closure, which may results in IH with higher rate of bowel strangulation; (2) the increased operative time; and (3) potential complications related to this suture, such as mesenteric hematomas and bowel kinking.

Even if mesenteric defect closure is recommended during RYGB, we decided not to close the Petersen's defect during OAGB. The rate of ischemia of the herniated bowel was low (9.3\%), and no resections were needed. However, patients with relapsing $\mathrm{IH}$ had smaller orifices and a higher rate of bowel ischemia (57\%). Our data are not in favor of a systematic closure of Petersen's defect during OAGB. We do not recommend systematic closure of the Petersen's space, in the lack of robust evidence on this topic. We highlight that this represents the authors' opinion based only on their personal experience. Further studies, ideally randomized studies about IH after OAGB, comparing closure versus non-closure, should be encouraged to provide high-grade evidence.

\section{Limits}

The present study is limited by its single-center and retrospective design. However, several strengths of the study should be highlighted: the remarkable number of enrolled patients (considering the rarity of the disease), and the new information on this disease that is provided.

\section{Conclusion}

The incidence of IH treated by surgical repair was $2.8 \%$ after OAGB without closure of the Petersen's defect, in a series including a total of 3368 patients. The rate of intestinal 
resection was null, the rate of bowel reversible ischemia was low, and patients had a low rate of postoperative complications and a short hospital stay.

Funding Open access funding provided by Università degli Studi di Roma La Sapienza within the CRUI-CARE Agreement.

\section{Declarations}

Conflict of Interest The authors declare no competing interests.

Ethics Approval All procedures performed in studies involving human participants were in accordance with the ethical standards of the institutional and/or national research committee and with the 1964 Helsinki declaration and its later amendments or comparable ethical standards.

Consent to participate Informed consent was obtained from all individual participants included in the study.

Open Access This article is licensed under a Creative Commons Attribution 4.0 International License, which permits use, sharing, adaptation, distribution and reproduction in any medium or format, as long as you give appropriate credit to the original author(s) and the source, provide a link to the Creative Commons licence, and indicate if changes were made. The images or other third party material in this article are included in the article's Creative Commons licence, unless indicated otherwise in a credit line to the material. If material is not included in the article's Creative Commons licence and your intended use is not permitted by statutory regulation or exceeds the permitted use, you will need to obtain permission directly from the copyright holder. To view a copy of this licence, visit http://creativecommons.org/licenses/by/4.0/.

\section{References}

1. Amor IB, Kassir R, Debs T, et al. Impact of mesenteric defect closure during laparoscopic Roux-en-Y gastric bypass (LRYGB): a retrospective study for a total of 2093 LRYGB. Obes Surg. 2019;29:3342-7.

2. Brammerloo YGA, Vannijvel M, Devriendt S, et al. Internal hernia after laparoscopic gastric bypass without preventive closure of mesenteric defects: a single institution's experience. J Gastrointest Surg. 2020; https://doi.org/10.1007/s11605-020-04761-w.

3. Quezada N, León F, Jones A, et al. High frequency of internal hernias after Roux-en-Y gastric bypass. Obes Surg. 2015;25:61521.

4. Al-Mansour MR, Mundy R, Canoy JM, et al. Internal hernia after laparoscopic antecolic Roux-en-Y gastric bypass. Obes Surg. 2015;25:2106-11.

5. Chowbey P, Baijal M, Kantharia NS, et al. Mesenteric defect closure decreases the incidence of internal hernias following laparoscopic Roux-en-Y gastric bypass: a retrospective cohort study. Obes Surg. 2016;26(9):2029-34.

6. Fabozzi M, Brachet Contul R, Millo P, et al. Intestinal infarction by internal hernia in Petersen's space after laparoscopic gastric bypass. World J Gastroenterol. 2014;20(43):16349-54.

7. Stenberg E, Szabo E, Ågren G, et al. Closure of mesenteric defects in laparoscopic gastric bypass: a multicentre, randomised, parallel, open-label trial. Lancet Lond Engl. 2016;387:1397-404.
8. Stenberg E, Ottosson J, Szabo E, et al. Comparing techniques for mesenteric defects closure in laparoscopic gastric bypass surgery-a register-based cohort study. Obes Surg. 2019;29:1229-35.

9. Magouliotis DE, Tzovaras G, Tasiopoulou VS, et al. Closure of mesenteric defects in laparoscopic gastric bypass: a meta-analysis. Obes Surg. 2020;30:1935-43.

10. Hajibandeh S, Hajibandeh S, Abdelkarim M, et al. Closure versus non-closure of mesenteric defects in laparoscopic Roux-en-Y gastric bypass: a systematic review and meta-analysis. Surg Endosc. 2020;34:3306-20.

11. Petrucciani N, Ciangura C, Debs T, et al. Management of surgical complications of previous bariatric surgery in pregnant women. A systematic review from the BARIA-MAT Study Group. Surg Obes Relat Dis. 2020;16:312-31. https://doi.org/10.1016/j.soard.2019. 10.022 .

12. Moore KA, Ouyang DW, Whang EE. Maternal and fetal deaths after gastric bypass surgery for morbid obesity. N Engl J Med. 2004;351:721-2.

13. Welbourn R, Hollyman M, Kinsman R, et al. Bariatric surgery worldwide: baseline demographic description and one-year outcomes from the Fourth IFSO Global Registry Report 2018. Obes Surg. 2019;29(3):782-95.

14. Parmar CD, Mahawar KK. One anastomosis (mini) gastric bypass is now an established bariatric procedure: a systematic review of 12 , 807 patients. Obes Surg. 2018;28:2956-67.

15. Carbajo MA, Luque-de-León E, Jiménez JM, et al. Laparoscopic one-anastomosis gastric bypass: technique, results, and long-term follow-up in 1200 Patients. Obes Surg. 2017;27:1153-67.

16. Haddad A, Fobi M, Bashir A, et al. Outcomes of one anastomosis gastric bypass in the IFSO Middle East North Africa (MENA) Region. Obes Surg. 2019;29:2409-14.

17. Musella M, Apers J, Rheinwalt K, et al. Efficacy of bariatric surgery in type 2 diabetes mellitus remission: the role of mini gastric bypass/one anastomosis gastric bypass and sleeve gastrectomy at 1 year of follow-up. A European survey. Obes Surg. 2016;26:93340.

18. De Luca M, Tie T, Ooi G, et al. Mini gastric bypass-one anastomosis gastric bypass (MGB-OAGB)-IFSO Position Statement. Obes Surg. 2018;28:1188-206.

19. Facchiano E, Iannelli A, Lucchese M. Internal hernia after minigastric bypass: Myth or reality? J Visc Surg. 2016;153:231-2.

20. Liagre A, Debs T, Kassir R, et al. One anastomosis gastric bypass with a biliopancreatic limb of $150 \mathrm{~cm}$ : weight loss, nutritional outcomes, endoscopic results, and quality of life at 8-year follow-up. Obes Surg. 2020;30:4206-17. https://doi.org/10.1007/s11695-02004775-y.

21. Frøkjær JB, Jensen WN, Holt G, et al. The diagnostic performance and interrater agreement of seven CT findings in the diagnosis of internal hernia after gastric bypass operation. Abdom Radiol N Y. 2018;43:3220-6.

22. Ederveen JC, van Berckel MMG, Nienhuijs SW, et al. Predictive value of abdominal $\mathrm{CT}$ in evaluating internal herniation after bariatric laparoscopic Roux-en-Y gastric bypass. Br J Surg. 2018;105: 1623-9.

23. Clavien PA, Barkun J, de Oliveira ML, et al. The Clavien-Dindo classification of surgical complications: five-year experience. Ann Surg. 2009;250:187-96.

24. Kular KS, Prasad A, Ramana B, et al. Petersen's hernia after mini (one anastomosis) gastric bypass. J Visc Surg. 2016;153:321.

25. Kermansaravi M, Kazazi M, Pazouki A. Petersen's space internal hernia after laparoscopic one anastomosis (mini) gastric bypass. Case Rep Surg. 2018;2018:9576120.

26. Abbas A, Kermansaravi M, Eghbali F, et al. Another Petersen's space hernia after one anastomosis gastric bypass: a video case report. Obes Surg. 2019;29:3776-7. 
27. AlZarooni N, Abou Hussein B, Al Marzouqi O, et al. Gastric remnant perforation caused by Peterson's hernia following one anastomosis gastric bypass: a rare complication. Obes Surg. 2020;30: 3229-32.

28. Facchiano E, Soricelli E, Lucchese M. Laparoscopic management of internal hernia after one anastomosis gastric bypass (OAGB). Obes Surg. 2020;30:4169-70. https://doi.org/10.1007/s11695020-04791-y.

29. Liagre A, Queralto M, Juglard G, et al. Multidisciplinary Management of leaks after one-anastomosis gastric bypass in a single-center series of 2780 consecutive patients. Obes Surg. 2019;29:1452-61.

30. Kassir R, Petrucciani N, Debs T, et al. Conversion of one anastomosis gastric bypass (OAGB) to Roux-en-Y gastric bypass
(RYGB) for biliary reflux resistant to medical treatment: lessons learned from a retrospective series of 2780 consecutive patients undergoing OAGB. Obes Surg. 2020;30:2093-8.

31. Nuytens F, D'Hondt M, Van Rooy F, et al. Closure of mesenteric defects is associated with a higher incidence of small bowel obstruction due to adhesions after laparoscopic antecolic Roux-en-Y gastric bypass: A retrospective cohort study. Int J Surg Lond Engl. 2019;71:149-55.

Publisher's Note Springer Nature remains neutral with regard to jurisdictional claims in published maps and institutional affiliations. 